Regular Articles

\title{
SPR optimization using metamaterials in a D-type PCF refractive index sensor
}

\author{
D.F. Santos ${ }^{\mathrm{a}, \mathrm{c}, *}$, A. Guerreiro ${ }^{\mathrm{b}, \mathrm{c}}$, J.M. Baptista ${ }^{\mathrm{a}, \mathrm{c}}$ \\ ${ }^{a}$ Faculdade de Ciências Exatas e da Engenharia, Universidade da Madeira, Funchal 9000, Portugal \\ ${ }^{\mathrm{b}}$ Faculdade de Ciências da Universidade do Porto, 4169, Portugal \\ ${ }^{\mathrm{c}}$ INESC TEC, Porto 4200, Portugal
}

\section{A R T I C L E I N F O}

Article history:

Received 25 August 2016

Accepted 6 November 2016

Available online 18 November 2016

\section{Keywords:}

Optical fiber sensor

Refractive index sensor

D-type photonic crystal fiber

Surface plasmon resonance

Metamaterials

COMSOL multiphysics

\begin{abstract}
A B S T R A C T
Using the finite element method (FEM), this paper presents a numerical investigation of the performance analysis of a D-type photonic crystal fiber (D-type PCF) for refractive index sensing, based on surface plasmon resonance (SPR) with a planar structure made out of a metamaterial. COMSOL Multiphysics was used to evaluate the design of the referred refractive index optical fiber sensor, with higher accuracy and considerable economy of time and resources. A study of different metamaterials concentrations conformed by aluminum oxide $\left(\mathrm{Al}_{2} \mathrm{O}_{3}\right)$ and silver $(\mathrm{Ag})$ is carried out. Another structural parameters, which influences the refractive index sensor performance, the thickness of the metamaterial, is also investigated. The results indicate that the use of metamaterials provides a way of improving the performance of SPR sensors on optical fibers and allows to tailor the working parameters of the sensor.
\end{abstract}

(c) 2016 Elsevier Inc. All rights reserved.

\section{Introduction}

In the past decade, resonant interaction between surface plasmons and light in metal films have introduced a revolution in high sensitivity optical sensing. Nowadays, with the development of improved fabrication techniques of metal-dielectric nanostructures it is possible to develop new artificial materials, such as optical metamaterials. These materials combine the properties of their constituent materials, and open new ways to explore the interaction between light and matter [1], and in particular between light and surface plasmons. In this paper, we address the use of metamaterial films as an alternative to metal films in SPR optical fiber sensors.

Surface plasmons generate exceptionally robust and spectrally narrowed optical fields far beyond the diffraction limit, which are strongly dependent on geometric parameters of the films [2] (such as thickness) as well as the refractive index of the media surrounding the metal [3]. These fields can couple the optical fields guided inside a waveguide with the exterior, thus imprinting their spectral features and dependencies in the optical losses of the fiber. This can be used to strongly improve the performance of optical fiber sensors. Indeed, many configurations of fiber optic sensors are

\footnotetext{
* Corresponding author at: Faculdade de Ciências Exatas e da Engenharia, Universidade da Madeira, Funchal 9000, Portugal.

E-mail address: diegonobregasantos@gmail.com (D.F. Santos).
}

based on SPR by removing the cladding (partial or totally) to allow the deposition of a thin metallic layer (usually gold or silver) [1]. This film supports the excitation of SPR and their interaction with the electromagnetic modes of the core.

There is an abundant work in literature that study different metallic layers to optimize SPR sensors (from controlling losses, to increasing sensor endurance, among others) [4-6]. The possible combinations of the material layers used are not restricted to metals. Also materials with high refractive index (HRI) can be used to control optical losses and the sensor operation range [7]. The main problem with these materials or this combination of the materials is the difficult control in the fabrication of the material films (including their thickness and roughness) and consequently in the control of their optical properties [8].

More recent works on SPR sensors, that combine different materials, have begun to explore the use of optical metamaterials. These artificial materials combine two or more materials to create a new artificial material which exhibits optical properties that cannot be found in naturally occurring materials and which can be designed to attain predefined performances [9]. These properties depend both on the optical properties of their constituent materials, their relative abundance and the geometry of the nanostructures used to combine them. This wide freedom available during fabrication allows to develop metamaterials with almost customized optical properties that can be used to optimize the performance of the sensor (e.g. loss, the light wavelength range, 
the refractive index range detection, among others) to values beyond that of conventional SPR sensors [10].

In this paper, we pursue this approach and present a sensor based on SPR that combines a D-type PCF fiber with a metamaterial $\left(\mathrm{Al}_{2} \mathrm{O}_{3}-\mathrm{Ag}\right)$. The performance of the sensor is investigated through simulations based on FEM using COMSOL Multiphysics [7]. The sensor analysis is focused on the percentage of each constituent of the metamaterials and the layer thickness, which are the main parameters in determining their optical properties. The numerical simulation of new sensing concepts and configurations allows to select the ones with best performance to be fabricated, thus leading to a considerable economy of time and resources when compared to an exhaustive fabrication and test of all possible designs.

\section{Design and model}

We consider the design of an optical sensor of refractive index composed of a PCF fiber with D-type profile, as described in Fig. 1 . The fiber is composed by a glass core with refractive index $n_{g}$, surrounded by an array of dielectric structures (corresponding to the holes in the PCF fiber) with refractive index equal to 1 . The refractive index $n_{g}$ is calculated using the Sellmeier equation [7]. It is assumed that the space outside the fiber is filled with the analytic medium to be studied, and having an external refractive index $n_{\text {ext }}$. The distance between the center of the fiber and the metamaterial layer is denoted by $d$ - residual cladding, the thickness of metamaterial layer by $d_{m}$, the diameter of the holes by $d_{\text {hole }}$, and the separation of the holes by $\Lambda$ - pitch.

PCF favors the confinement of light in the core, whereas the Dtype profile of the fiber promotes the interaction of the core modes with the external medium. The interaction of the electromagnetic modes with the external media is mediated by a metallic film that supports surface plasmons, which improves the sensitivity of the sensor [11]. In this paper we replace the metal with a metamaterial film composed of thin layers (sub-wavelength) of silver and alumina (see the inset of Fig. 1).

The optical properties of the metamaterial depend on the relative thickness of the silver and alumina layers. The effective refractive index of the metamaterial film can be calculated using an effective medium approach, of which the Maxwell- Garnett Theory (MGT) and the Bruggeman effective medium theory (BEMT) are the most commonly used models [9]. In our case, we adopt the BEMT since it is more adequate when the two material have quite distinct permittivity, rather than the MGT, which considers only materials with similar permittivity,

$f_{1} \frac{\epsilon_{1}-\epsilon_{m}}{\epsilon_{1}+v \epsilon_{m}}+f_{2} \epsilon_{2}-\epsilon_{m} \epsilon_{2}+v \epsilon_{m}=0$

where $\varepsilon_{m}$ is the dielectric constant of the metamaterial, $\varepsilon_{i}(i=1,2)$ is the refractive index of each of the two types of inclusions ( $\mathrm{Ag}$ and $\mathrm{Al}_{2} \mathrm{O}_{3}$, respectively), with volume filling-ratios of $f_{1}$ and $f_{2}=1-f_{1}$, respectively, and $\eta$ is the form factor ( $\eta=2$ for this geometry). Other nanostructures of $\mathrm{Al}_{2} \mathrm{O}_{3}-\mathrm{Ag}$ can also be used, including silver nanowires or nanorods immersed in an alumina matrix. In these cases, the same homogenization model can be used by replacing the form factor $\eta$ with the appropriate value according to the geometry of the nanostructures. The general optical properties obtained for other metamaterials are alike and therefore we focus here on the simplest form to fabricate [10].

The complex dielectric of the metamaterial results from the combination of properties from its metal and dielectric components. As a result, the metamaterial layer can be considered as an artificial metal layer capable of supporting effective plasmonic modes. Using Eq. (1) we can calculate the complex dielectric of the metamaterial as shown in Fig. 2, for different concentrations of Ag.

Fig. 2 shows the real (Fig. 2a) and imaginary (Fig. 2b) parts of the dielectric for the different concentrations or fill ratios of the $\mathrm{Ag}$ and $\mathrm{Al}_{2} \mathrm{O}_{3}$ calculated using the BEMT model. Like in the case of a pure silver film, the metamaterial films with fill ratios up to $50 \%$ have a negative dielectric constant for the large majority of the spectral range considered, which is a necessary condition to support surface plasmons. In the case of a fill ration of $50 \%$, the metamaterial is close to a epsilon-near-zero regime, situation where the effective medium approach may have a limited validity.

The study is based on the calculation of the guided modes taking into account both the PCF D-type fiber and the metamaterial layer. These modes are referred as supermodes (SMs) since they correspond to the hybridization of the individual modes of each of the structures that compose the device taken isolated, which in our case are the fundamental guided mode of the fiber and the plasmon modes in the metal or metamaterial layer. All these modes can be calculated numerically by solving the wave equation for the Fourier components of the electric field [12]

$\nabla \times\left[\nabla \times E(\gamma, \omega)-k_{0}^{2} \tilde{\epsilon}_{\gamma}(\gamma, \omega)\right] E(\gamma, \omega)$

where $\omega$ is the frequency, $E(r, \omega)$ is the electric field, $k_{0}=\omega / c$ is the wave-number of the field mode and $c$ is the speed of light. The term $\tilde{\varepsilon}_{r}$ represents the complex relative dielectric function written in terms of the real part $\left(n_{r}{ }^{\prime}\right)$ and imaginary part $\left(n_{r}{ }^{\prime}\right)$ of the refractive index. The computer model is based on FEM implemented in COMSOL Multiphysics, following the method described in a previous work [13].

The optical power flow of the guided mode is given by the real part of the time-averaged Poynting vector

$S=(1 / 2) \operatorname{Re}\left(e x h^{*}\right) e^{-o z}$

whereas the sensor resolution $(R)$ is calculated from the sensitivity according to [14]

$R(\lambda)=\frac{\Delta n_{\text {ext }} \Delta \lambda_{\text {min }}}{\Delta \lambda_{\text {peak }}}$

where $\alpha=2 n_{e f}$ " $k_{0}$ is known as the power absorption coefficient, $\lambda_{\text {min }}$ is the minimum value associated with an experimental accurately

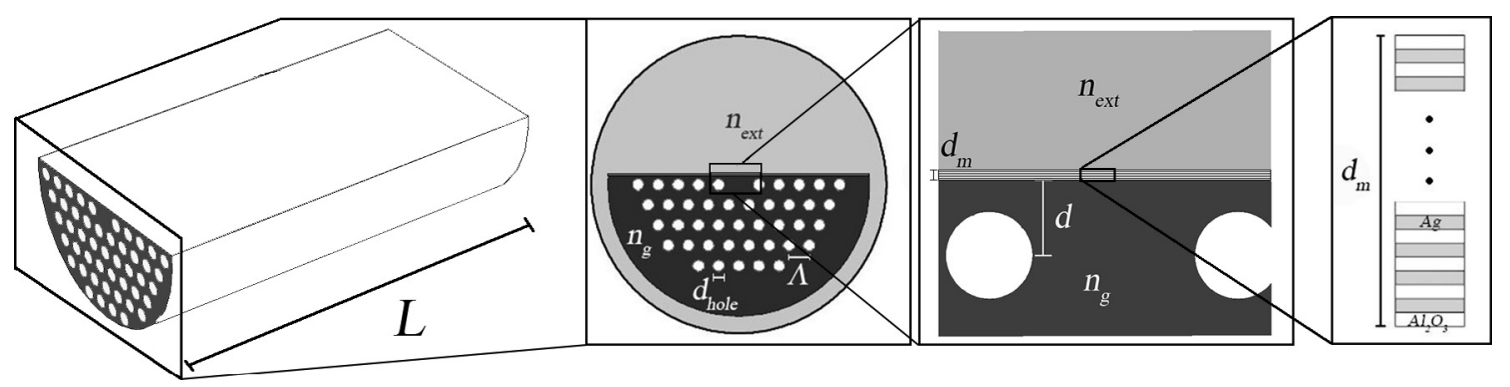

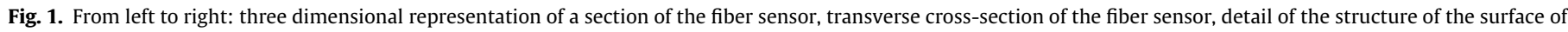
the sensor indicating the position metamaterial film and a scheme of the thin metal-dielectric layers that compose the metamaterial. 

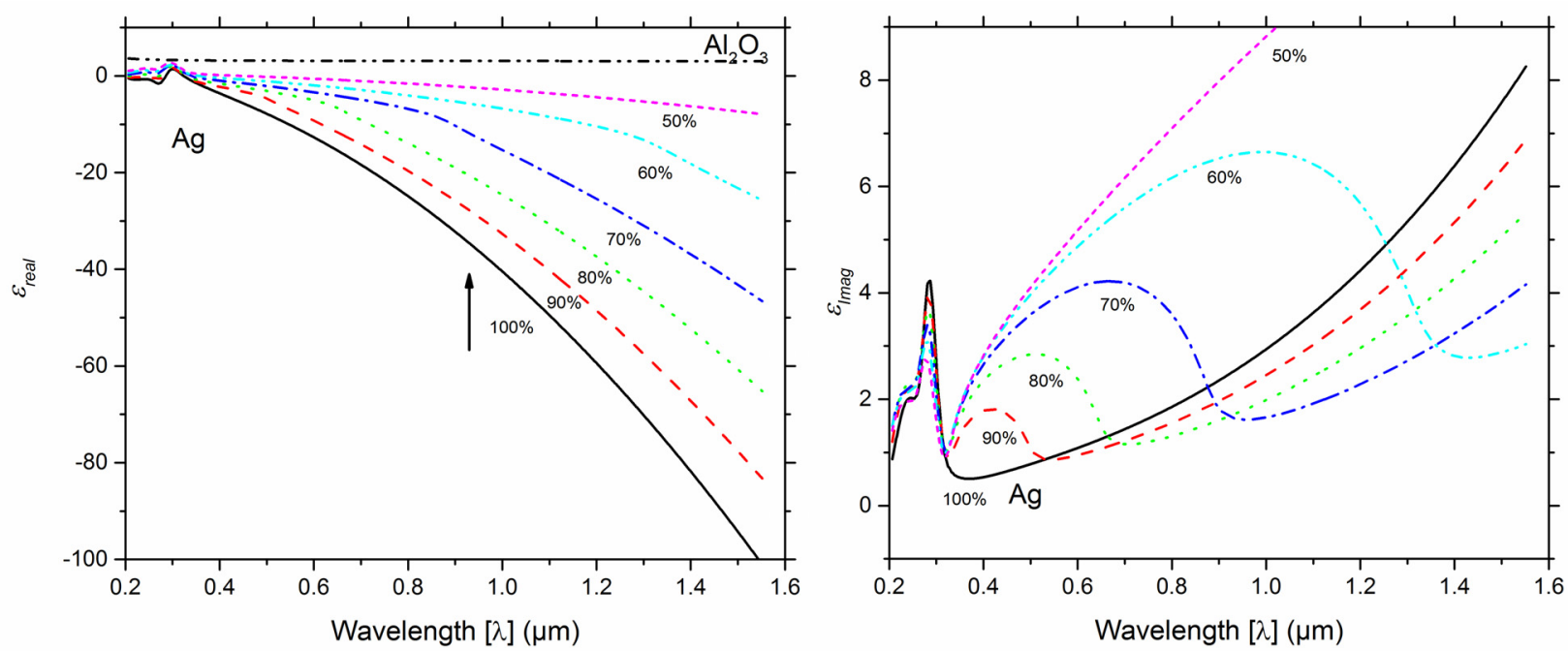

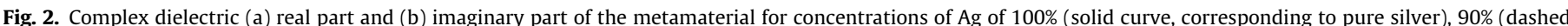

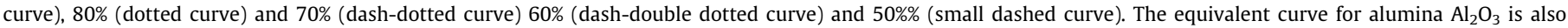
presented for reference (small dotted curve).

spectral variation detection and $\Delta \lambda_{\text {peak }}$ is the peak wavelength of the resonance obtained in the simulations for different values of external refractive index $\left(n_{\text {ext }}\right)$.

\section{Results}

\subsection{Metamaterial versus pure metal layers}

In this section we compare the conventional sensor based on a PCF D-type and a metal layer (e.g. Ag) with the version based on a metamaterial with $20 \%$ of $\mathrm{Al}_{2} \mathrm{O}_{3}$ and $80 \%$ of Ag using Eq. (2) to compute losses per $\mathrm{mm}$, as function of the wavelength, for different values of the external refractive index. The results are shown in Fig. 3 where it is possible to identify a clear peak in losses, typical of the SPR, which is not only strongly affected by the change in external refractive index, but also is dramatically affected by the replacement of the metal with a metamaterial layer. Indeed, these results show a red-shift of the peak in wavelength for higher external refractive index. But what is relevant is that not only the values of the losses for metamaterials are considerably higher amounting to $40 \mathrm{~dB} / \mathrm{mm}$ (instead of $10 \mathrm{~dB} / \mathrm{mm}$ obtained with a pure metal layer) but also the operation range in wavelength is around $700 \mathrm{~nm}$ (instead of $550 \mathrm{~nm}$ obtained with a pure metal layer) for $n_{\text {ext }}=1.30$. This suggests that metamaterials allow a stronger inter- (a)

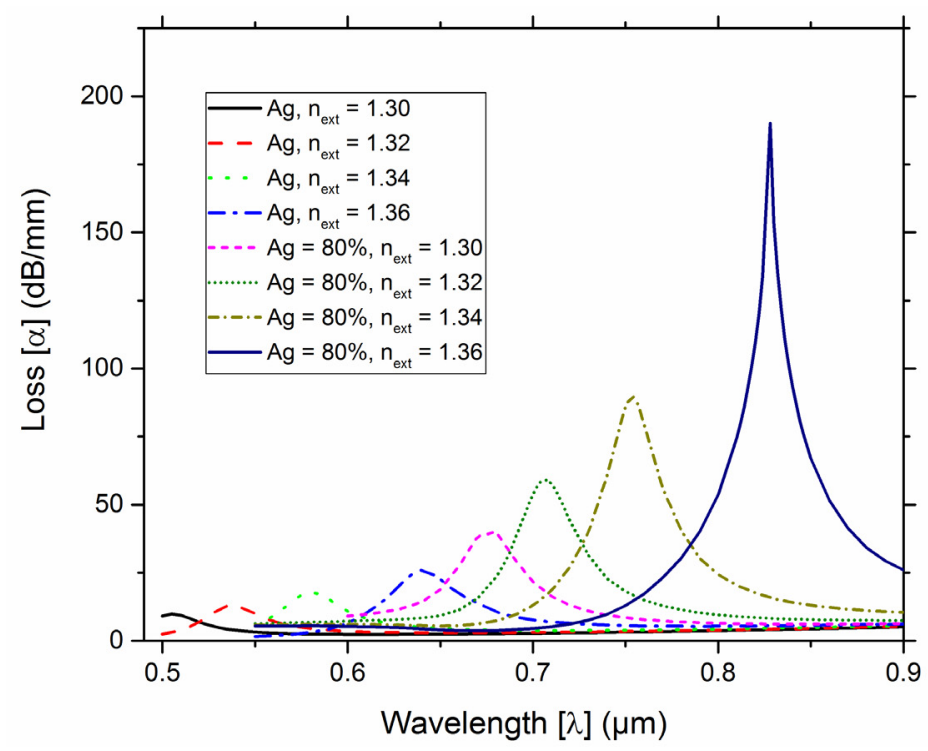

(b)

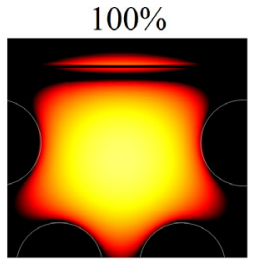

$0.550 \mu \mathrm{m}$

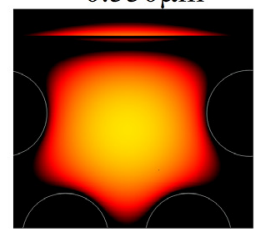

$0.580 \mu \mathrm{m}$

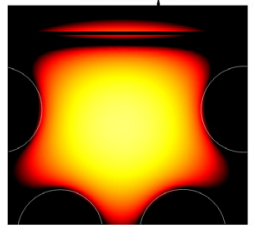

$0.650 \mu \mathrm{m}$

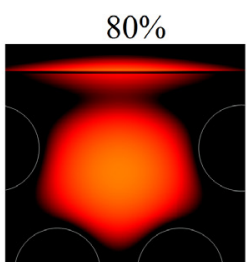

$0.700 \mu \mathrm{m}$

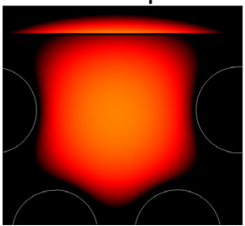

$0.750 u \mathrm{~m}$

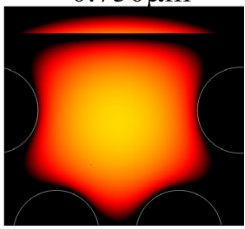

$0.800 \mu \mathrm{m}$

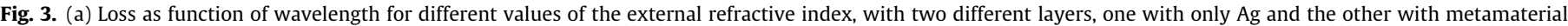

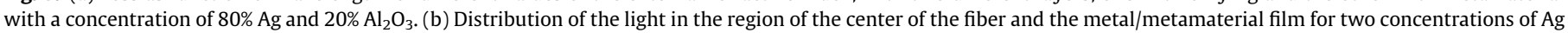

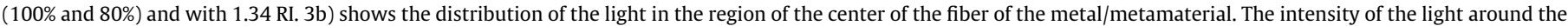

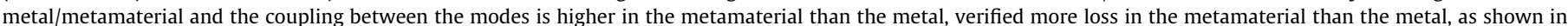
Fig. 3a. 
action between light in the fiber and the external medium at higher wavelengths, closer to the $850 \mathrm{~nm}$ optical window and therefore are more adequate for integration in telecom optical fiber networks. It also follows from these results that it is possible to optimize and balance the ranges of operation of the sensor in both wavelength and external refractive index by choosing the relative concentration of the metal in the metamaterial and the layer thickness. For example, if the sensor is to work between $600 \mathrm{~nm}$ and $700 \mathrm{~nm}$ and measure refractive indexes between 1.30 and 1.32 RIU instead of 1.35 and 1.36 RIU, then it is possible to fine tune the concentration of $\mathrm{Ag}$ around $80 \%$ for optimal performance.

Considering a spectral variation of $0.1 \mathrm{~nm}$ that can be accurately detected experimentally and using Eq. (3), the values of the sensitivity, sensor resolution and spectral range of the refractive index sensor with the considered metamaterial, for different ranges of variation of the external refractive index are shown in Table 1. We also present the equivalent results for a sensor with a layer of pure metal. Clearly, the sensor with the metamaterial outper- forms its counterpart with pure metal in all parameters even though the gains in resolution are marginal. As expected from Fig. 3, the major improvements are in terms of sensitivity (over $30 \%$ ) and in wavelength operation range, especially for variation of the refractive index in the range of $[1.34,1.36]$ where the sensor can actually operate at $830 \mathrm{~nm}$.

\subsection{Loss for different concentrations of $\mathrm{Al}_{2} \mathrm{O}_{3}$ and $\mathrm{Ag}$}

The previous section demonstrated the best performance of metamaterial sensors when compared with their metal counterpart, and the increased freedom in designing the sensor associated with control of the concentrations of the different materials that compose the metamaterial, as well as the layer thickness. This section addresses the characterization of the sensor in terms of the first parameter. Fig. 4 and Table 2 show the light loss through the sensor as function of the wavelength for metamaterials with different concentrations of $\mathrm{Ag}-\mathrm{Al}_{2} \mathrm{O}_{3}$. The results show a

Table 1

Sensitivity, resolution and range of the wavelength values for the refractive index optical fiber sensors based on two materials (Ag and metamaterial).

\begin{tabular}{|c|c|c|c|c|c|c|}
\hline \multirow[t]{2}{*}{ Range of $n_{e x t}$} & \multicolumn{2}{|c|}{ Sensitivity (nm/RIU) } & \multicolumn{2}{|c|}{ Resolution (RIU) } & \multicolumn{2}{|c|}{ Range $\lambda(\mathrm{nm})$} \\
\hline & $\mathrm{Ag}$ & Metamaterial & $\mathrm{Ag}$ & Metamaterial & $\mathrm{Ag}$ & Metamaterial \\
\hline$[1.30,1.32]$ & 1750 & 2300 & $5.88 \times 10^{-5}$ & $4.35 \times 10^{-5}$ & $500-530$ & $670-710$ \\
\hline$[1.32,1.34]$ & 2000 & 2400 & $4.76 \times 10^{-5}$ & $4.17 \times 10^{-5}$ & $530-580$ & $710-755$ \\
\hline$[1.34,1.36]$ & 2800 & 3700 & $3.57 \times 10^{-5}$ & $2.70 \times 10^{-5}$ & $580-640$ & $755-830$ \\
\hline
\end{tabular}

(a)

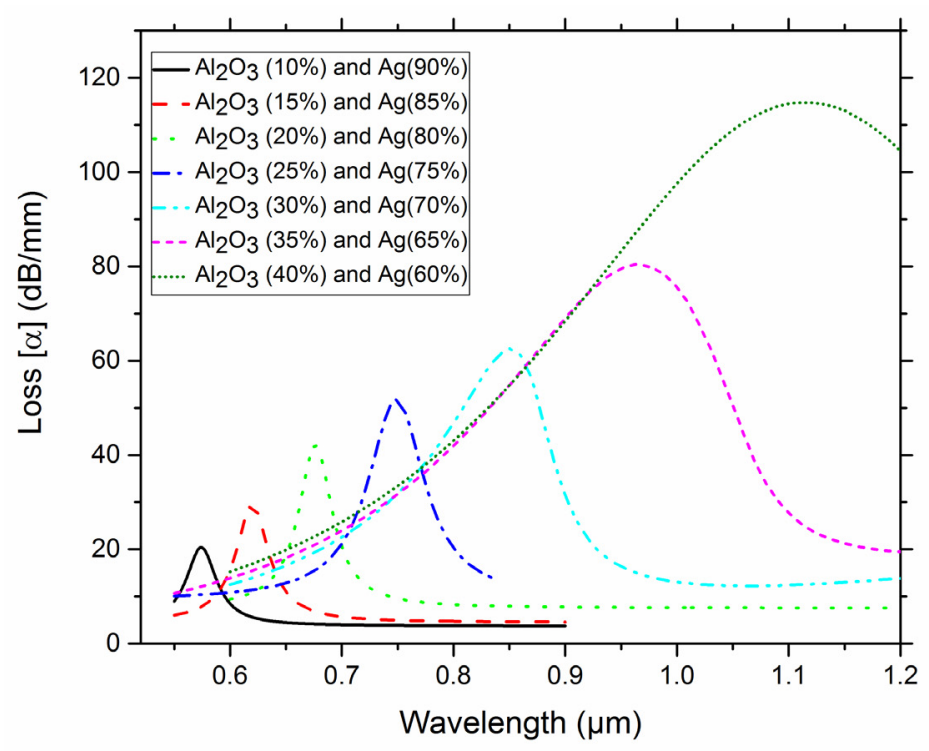

(b)

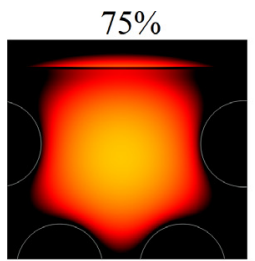

$0.720 \mu \mathrm{m}$

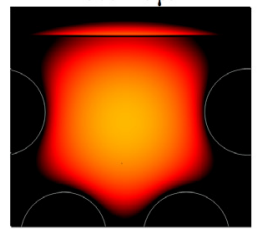

$0.740 \mu \mathrm{m}$

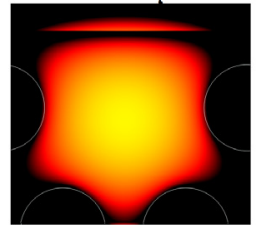

$0.800 \mu \mathrm{m}$

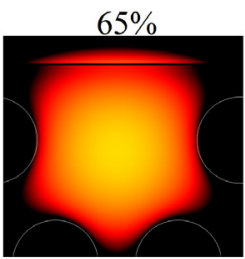

$0.900 \mu \mathrm{m}$

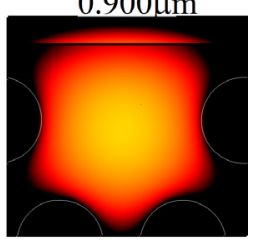

$0.960 \mu \mathrm{m}$

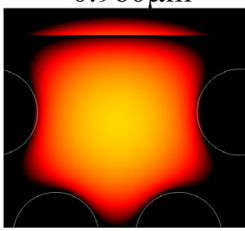

$1.040 \mu \mathrm{m}$

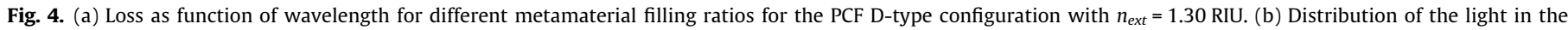
region of the center of the fiber and the metamaterial film for two concentrations of $\mathrm{Ag}$ ( $75 \%$ and $65 \%$ ).

Table 2

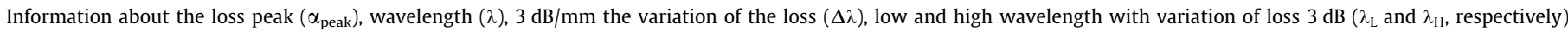
information about narrow of the sensor, extracted from Fig. 4.

\begin{tabular}{|c|c|c|c|c|c|c|}
\hline Concentration & $\alpha_{\text {peak }}(\mathrm{dB} / \mathrm{mm})$ & $\lambda(\mathrm{nm})$ & $\Delta \lambda(\mathrm{dB} / \mathrm{mm})$ & $\lambda_{\mathrm{L}}(\mathrm{nm})$ & $\lambda_{\mathrm{H}}(\mathrm{nm})$ & $\lambda_{\mathrm{H}}-\lambda_{\mathrm{L}}(\mathrm{nm})$ \\
\hline $\mathrm{Al} 2 \mathrm{O} 3 \mathrm{10} \%$ and $\mathrm{Ag} 90 \%$ & 20.422 & 574 & 17.422 & 566.47 & 581.88 & 15.41 \\
\hline $\mathrm{Al} 2 \mathrm{O} 320 \%$ and $\mathrm{Ag} 80 \%$ & 41.966 & 676 & 38.966 & 670.87 & 682.09 & 11.22 \\
\hline $\mathrm{Al} 2 \mathrm{O} 330 \%$ and $\mathrm{Ag} 70 \%$ & 62.619 & 850 & 59.619 & 834.40 & 864.10 & 29.70 \\
\hline $\mathrm{Al} 2 \mathrm{O} 340 \%$ and $\mathrm{Ag} 60 \%$ & 114.730 & 1115 & 111.730 & 1069.00 & 1159.91 & 90.91 \\
\hline
\end{tabular}


red-shift in wavelength of the resonance peak for decreasing concentrations of $\mathrm{Ag}$ similar to those reported in previous studies [7].

From Table 2 we conclude that metamaterials with higher concentrations of $\mathrm{Ag}$ produce narrower resonance peaks, an effect which is especially noticeable for Ag concentration above of $70 \%$. This puts a lower limit to the concentration of $\mathrm{Ag}$, since below this value one obtains wide resonance peaks and strong losses, thus resulting in feeble optical signal and low signal to noise ratios. The best sensing performance occurs for a metamaterial with $20 \%$ of $\mathrm{Al}_{2} \mathrm{O}_{3}$ and $80 \%$ the $\mathrm{Ag}$, where the resonance peak is relatively narrow and losses are not excessive. The transition between a narrow and broad resonance identifiable in the loss spectrum described in Fig. 4a for metamaterial with increased $\mathrm{Al}_{2} \mathrm{O}_{3}$ concentrations can be explained in terms of the imaginary part of the dielectric constant illustrated in Fig. 2b. For pure silver, the imaginary part for wavelengths above $0.3 \mu \mathrm{m}$ grows monotonically, resulting in a narrow SPR resonance at lower wavelengths. For metamaterial with higher concentrations of $\mathrm{Al}_{2} \mathrm{O}_{3}$, we notice the existence of a feature similar to a concave protuberance in the spectrum of the imaginary part of the dielectric constant, which allows more wavelengths to be near SPR resonance, thus broadening the loss peak observed in figure Fig. 4a and blue shifting it.

Fig. 4b shows the distribution of the light in the region of the center of the fiber and the metamaterial film for two concentrations of $\mathrm{Ag}$ (75\% and 65\%). The behavior of the light for both concentrations is similar, but the wavelength in the transition of the light occurs in a different wavelength range, which is in accordance with respective spectral profile of Fig. $4 \mathrm{a}$.

\subsection{Loss for different thicknesses of the metamaterial}

In this section we address the characterization of the metamaterial sensor in terms of the layer thickness. The metamaterial thickness is strongly constrained not only by fabrication limitations, but also by the validity of the homogenization model used to compute the optical properties of the metamaterial (see Eq. (1) and the fill ratio $f$ ) and ultimately by the physical limitations of the nanoscale components of the metamaterial to respond collectively to the optical field and behave as a single bulk synthetic optical material. For example, if we consider the layered medium chosen in this article it is not possible to have metamaterial layers of thicknesses of about a few atoms as it would in the case of a pure metal. Indeed, in such case it would be impossible to pile up sufficient layers of both $\mathrm{Ag}$ and $\mathrm{Al}_{2} \mathrm{O}_{3}$ to obtain an intended value of relative concentration. This could however be overcome by considering other types of metamaterials, such as those composed of metal nanodisks embedded in a dielectric matrix [10] which would be able to cover the entire range of metal and dielectric concentrations, even for layers with atomic thicknesses, by adjusting the radius and average distance between the metal disks. Then the homogenization model would still be valid but with a different value of form factor $f$.

Fig. 5 describes the attenuation through the fiber as function of the wavelength for different values of the thickness of the metamaterial $\left(d_{m}\right)$ and shows a slight red-shift in wavelength when the thickness of the metal is increased, although for large thicknesses of the metal the shift is smaller than for short thicknesses. The decreased of the $d_{m}$ shows an increase of the intensity of the loss, phenomenon observed in pure metals [7]. This suggests that it is possible to do some fine tuning of the sensor performance (such as the operation range and the amount of losses) by adjusting the thickness of the metamaterial.

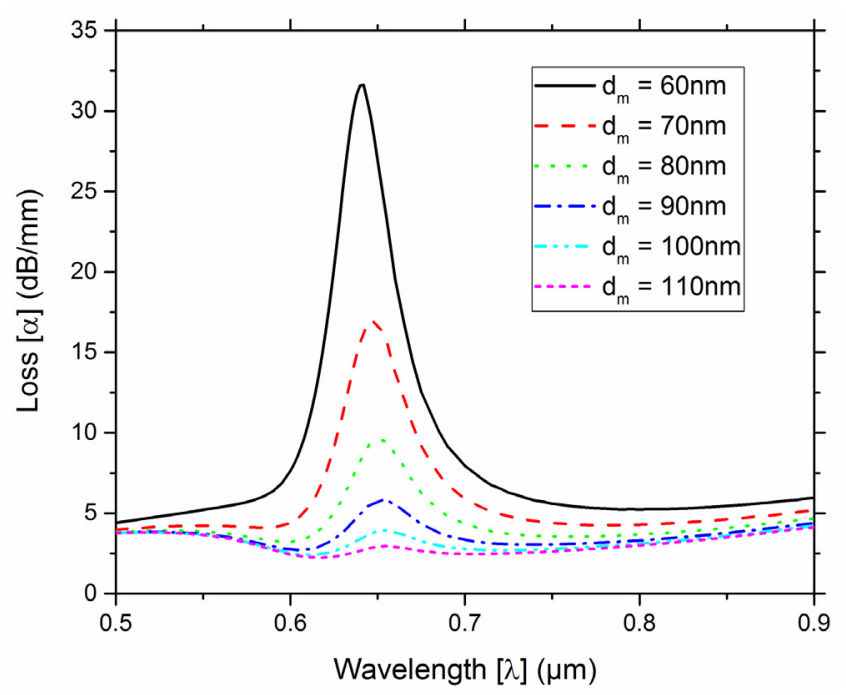

Fig. 5. Loss as function of wavelength for different thicknesses of the metamaterial PCF D-type configuration fiber with $n_{\text {ext }}=1.32 \mathrm{RIU}$ and $\mathrm{Ag}=90 \%$.

\subsection{Optimization of the sensor}

The two previous sections have shown that it is possible to improve the performance of the sensor by controlling both the concentration of $\mathrm{Ag}$ and thickness of the metamaterial. In this section we combined those results and discuss how they can be used to design a sensor with optimized performance. In particular, it was concluded that the increase in losses associated with decreasing the metal concentration could be partially compensated with an increase in layer thickness. On the other hand, both a decrease in metal concentration and an increase in layer thickness would contribute to a red shift of the peak towards the $850 \mathrm{~nm}$. These considerations suggest that the best design parameters to consider when optimizing the sensor performance are on one hand the layer thickness and on the other the amount of metal per area of the metamaterial film, rather than the actual concentration of metal. A good estimate of the amount of metal per area can be obtained by the product between the thickness of the layer and the concentration of $\mathrm{Ag}$ in the metamaterial, designated by $\sigma_{\mathrm{Ag}}=\% \mathrm{Ag} \times d_{m}$.

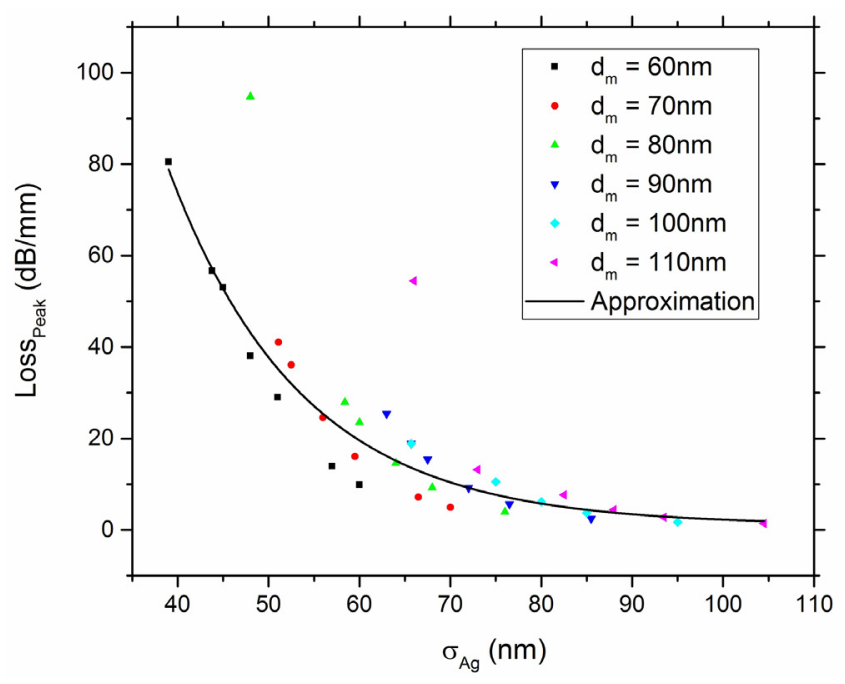

Fig. 6. The loss peak as function of the relative concentration and thickness of the metamaterial with $n_{\text {ext }}=1.30 \mathrm{RIU}$. 


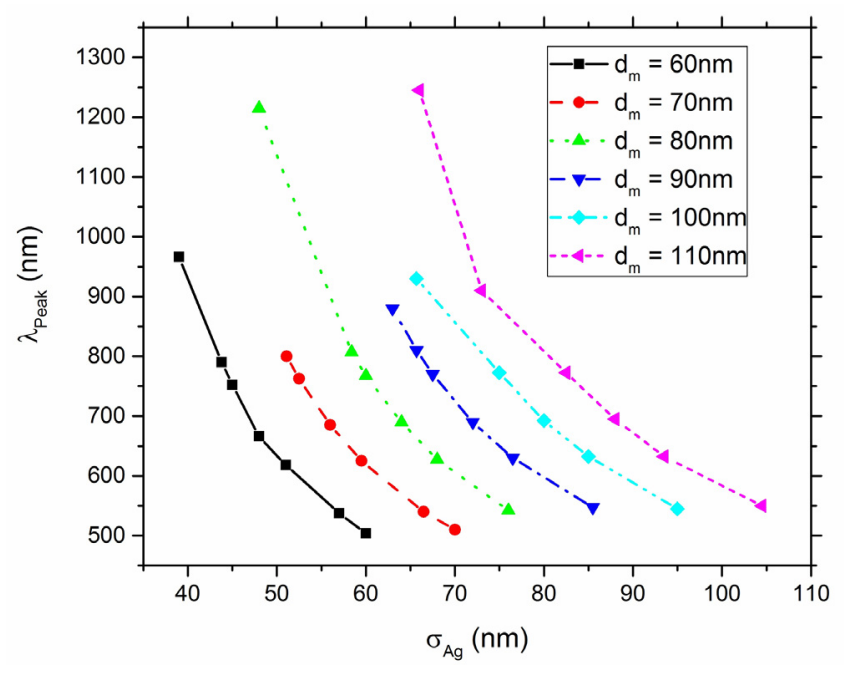

Fig. 7. The wavelength peak of the loss as function of the relative concentration and thickness of the metamaterial for $n_{\text {ext }}=1.30$.

In Fig. 6 we have plotted the amount of losses of the resonance peak for different values of the product between Ag concentration and thickness. Clearly, the wide majority of these points fall along the same trend line thus supporting our hypothesis. This shows that the relevant parameter is the total cross section between the optical field and the atoms of metal as the former crosses the layer. In other words, the losses are approximately determined by the amount of metal atoms that interact with the field per unit area and are somewhat independent of whether they are all concentrated in a single metal layer or distributed along the metamaterial. Therefore, the main effect of the metamaterial, when compared to a film of pure metal with the same amount of atoms per unit of surface (and hence with a different thickness) is to red shift the peak of the SPR resonance.

To verify this second conclusion, we plotted in Fig. 7 the peak wavelength as a function of $\sigma_{\text {Ag. }}$. In this case, the points corresponding to different thicknesses fall on similar, yet parallel, trend lines.

The conclusions resulted from Fig. 6 and Fig. 7 can now be used in the design and optimization of the sensor by allowing to choose the thickness of the metamaterial and concentration of $\mathrm{Ag}$ that yield the best performance for a given spectral range of operation, as well as, for a specific range of measurement of $n_{\text {ext }}$. For example, to measure an external refractive index around 1.30 RIU using a sensor operating around $700 \mathrm{~nm}$, it is possible to use Fig. 7 to select a metamaterial thickness of, for instance, $70 \mathrm{~nm}$ corresponding to $\sigma_{\mathrm{Ag}} \approx 55 \mathrm{~nm}$ and a concentration of the $\mathrm{Ag}$ of $79 \%=\sigma_{\mathrm{Ag}} / d_{m}=55,70$. The level of the losses at the peak can be estimated from Fig 6 to be about $30 \mathrm{~dB} / \mathrm{nm}$. For different ranges of $n_{\text {ext }}$, we need to consider the effect of the external refractive index in the sensor performance as illustrated in Fig. 4.

\section{Conclusions}

COMSOL Multiphysics was used to evaluate the design of an optical sensor of refractive index with higher accuracy and considerable economy of time and resources. The sensor design was composed of a PCF D-type fiber with metamaterial layer and demonstrated the possibility of controlling the losses and operation wavelength of the sensor over a wide range by changing the relative fraction of the different materials that compose the metamaterial.
From Table 2 we conclude that metamaterials with higher concentrations of $\mathrm{Ag}$ produce narrower resonance peaks, an effect which is especially noticeable for Ag concentration above $70 \%$. This puts a lower limit to the concentration of $\mathrm{Ag}$, as below this value, one obtains wide resonance peaks and strong losses, thus resulting in feeble optical signal and low signal to noise ratios. Fig. 6 and Fig. 7 permit to design and optimize the sensor by allowing to choose the thickness and concentration of $\mathrm{Ag}$ that yield the best performance for a given spectral range of operation, as well as, for a specific range of measurement of $n_{\text {ext }}$.

These results indicate that the use of metamaterials provides not only a way of improving the performance of SPR sensors on optical fibers, but also to adequately control and customize the performance characteristics of the sensor. In practice, the metamaterial works as an artificial metal with optical properties that cannot be found in natural materials and can be customized to design specifications of the sensor. The analysis of the results provides a method of optimization for choosing the best metamaterial parameters that can be applied in the engineering of this types of sensors.

\section{Acknowledgments}

This work was supported by National Funds through the FCT Fundação para a Ciência e a Tecnologia (Portuguese Foundation for Science and Technology) through the $\mathrm{PhD}$ grant SFRH/ $\mathrm{BD} / 85068 / 2012$ and as part of project UID/EEA/50014/2013, and also is financed by the ERDF - European Regional Development Fund through the Operational Programme for Competitiveness and Internationalisation - COMPETE 2020 Programme within project «POCI-01-0145-FEDER-006961».

\section{References}

[1] B. Lee, S. Roh, J. Park, Current status of micro- and nano-structured optical fiber sensors, Opt. Fiber Technol. 15 (2009) 209-221.

[2] H. Suzukia, M. Sugimotob, Y. Matsuic, J. Kondoha, Effects of gold film thickness on spectrum profile and sensitivity of a multimode-optical-fiber SPR sensor, Sens. Actuators, B 132 (2008) 26-33.

[3] S.-F. Wang, M.-H. Chiu, J.-C. Hsu, R.-S. Chang, F.-T. Wang, Theoretical analysis and experimental evaluation of D-type optical fiber sensor with a thin gold film, Optics Commun. 253 (2005) 283-289.

[4] K. Sharma, B.D. Gupta, On the performance of different bimetallic combinations in surface plasmon resonance based fiber optic sensors, J. Appl. Phys. 101 (2007) 093-111.

[5] K. Sharma, B.D. Gupta, Comparison of performance parameters of conventional and nano-plasmonic fiber optic sensors, Plasmonics 2 (2007) 51-54.

[6] K.-S. Lee, J.M. Son, D.-Y. Jeong, T.S. Lee, W.M. Kim, Resolution enhancement in surface plasmon resonance sensor based on waveguide coupled mode by combining a bimetallic approach, Sensors 10 (2010) 11390-11399.

[7] D.F. Santos, A. Guerreiro, J.M. Baptista, Numerical investigation of a refractive index SPR D-type optical fiber sensor using COMSOL multiphysics, Photonics Sensors 3 (2013) 61-66.

[8] P.R. West, I. Satoshi, V.N. Gururaj, K.E. Naresh, M.S. Vladimir, B. Alexandra, Searching for better plasmonic materials, Laser Photonics Rev. 808 (2010) $795-808$.

[9] W. Cai, V. Shalaev, Optical Metamaterials, Springer, New York, 2010.

[10] I.T. Leite, P. Fernandes, A. Hierro-Rodriguez, J.M. Teixeira, P.A.S. Jorge, A Guerreiro, Analysis of a fibre optic sensor design based on SPR in nanowire metamaterial films, in: Proc. SPIE 9157, 23rd International Conference on Optical Fibre Sensors, 9157, 1-4, 2014.

[11] M.A. Schmidt, L.N.P. Sempere, H.K. Tyagi, C.G. Poulton, P. St. J Russell, Waveguiding and plasmon resonances in two-dimensional photonic lattices of gold and silver nanowires, Phys. Rev. B 77 (2008) 033417.

[12] M. VFliziani, F. Maradei, Edge element analysis of complex configurations in presence of shields, IEEE Trans. Magn. 33 (1997) 1548-1551.

[13] D.F. Santos, A. Guerreiro, J.M. Baptista, SPR microstructured D-type optical fiber sensor configuration for refractive index measurement, IEEE Sens. J. 15 (2015) 5472-5477.

[14] Y. Lu, C.-J. Hao, B.-Q. Wu, X.-H. Huang, W.-Q. Wen, X.-Y. Fu, J.-Q. Yao Grapefruit fiber filled with silver nanowires surface plasmon resonance sensor in aqueous environments, Sensors 12 (9) (2012) 12016-12025. 